\title{
Factors Affecting the Prevalence of Gastro-oesophageal Reflux in Childhood Corrosive Oesophageal Strictures
}

\author{
Serdar H. İskit ${ }^{1}$, Zerrin Özçelik², Murat Alkan ${ }^{1}$, Selcan Türker ${ }^{3}$, Ünal Zorludemir ${ }^{1}$ \\ ${ }^{1}$ Department of Pediatric Surgery, Çukurova University Faculty of Medicine, Adana, Turkey \\ ${ }^{2}$ Department of Pediatric Surgery, Numune Hospital, Adana, Turkey \\ ${ }^{3}$ Department of Pediatric Surgery Unit, Şehit Kamil Hospital, Gaziantep, Turkey
}

Background: Gastro-oesophageal reflux may accompany the corrosive oesophageal damage caused by the ingestion of corrosive substances and affect its treatment. The factors that affect the development of reflux in these cases and their effects on treatment still remain unclear.

Aims: Our aim is to investigate the prevalence of gastro-oesophageal reflux in children with corrosive oesophageal strictures, the risk factors affecting this prevalence and the effects of gastro-oesophageal reflux on treatment.

Study Design: Case-control study.

Methods: We enrolled 52 patients with oesophageal stricture due to corrosive substance ingestion who were referred to our clinic between 2003 and 2010. Groups, which were determined according to the presence of gastro-oesophageal reflux (GER), were compared with each other in terms of clinical findings, results of examination methods, characteristics of the stricture and success of the treatment. Results: The total number of patients in our study was 52; 30 of them were male and 22 of them were female. The mean age of our study population was $4.2 \pm 2.88$ years. Thirty-three patients had gastrooesophageal reflux (63.5\%). Patients who had strictures caused by the ingestion of alkali substances were 1.6-times more likely to have reflux. There were no differences between patients with or without reflux in terms of number and localisation of strictures. Mean distance of stricture was longer in patients with reflux $(3.7 \pm 1.8 \mathrm{~cm})$ than in patients without $(2.2 \pm 1.0 \mathrm{~cm})(\mathrm{p}<0.005)$. Only one patient among 17 who had a long stricture $(\geq 4 \mathrm{~cm})$ did not suffer from reflux. Patients with long stricture were 1.9-times more likely to have reflux. Dilatation treatment was successful in $69.6 \%$ of patients with reflux and in $78.9 \%$ of patients without. The mean treatment period was $8.41 \pm 6.1$ months in patients with reflux and $8.21 \pm 8.4$ months in the other group. There was no significant difference between groups in terms of frequency of dilatation and dilator diameters $(\mathrm{p}>0.05)$.

Conclusion: Corrosive oesophageal stricture was usually accompanied by gastro-oesophageal reflux and the length of stricture is an important risk factor. Negative effects of reflux over dilatation treatment have not yet been demonstrated in the short-term. Nevertheless, this frequent rate of reflux may eventually increase the risk of oesophagitis and Barrett's oesophagus; therefore, we suggest that these effects should be prospectively evaluated in a large number of patients and these patients should be followed-up routinely in terms of the long-term effects of reflux.

(Balkan Med J 2014;31:137-42).

Key Words: Corrosive oesophageal burns, gastro-oesophageal reflux, oesophageal $\mathrm{pH}$ monitoring, oesophagitis
Oesophageal stricture is the most common complication of corrosive ingestion in childhood and standard dilatation treatment has a success rate of approximately 50-70\% (1-3). Clinical studies conducted on the achievement of dilatation treatment emphasise the effects of the following parameters: start of dilatation treatment, stricture length, largest dilatator diameter used in the dilatation program and development of any perforations during the dilatation process (4). With the exception of corrosive substance ingestion, negative effects of gastro-oesophageal reflux (GER) on other causes of oesophageal strictures are clearly known. Previous studies published in English have not discussed the details of the relationship between stricture formation and
GER, nor the risk factors which cause the development of GER in patients suffering from oesophageal stricture due to corrosive substance ingestion (5-9). The aim of this clinical study is to investigate the relating factors affecting the prevalence of GER and the effects of GER on the treatment of patients suffering from oesophageal stricture due to corrosive substance ingestion.

\section{MATERIAL AND METHODS}

Çukurova University Ethics Committee approved the study and written informed consent forms were signed by families of the par- 
ticipating patients. We retrospectively analysed the data of 52 patients who were treated for corrosive oesophageal stricture (CES) in our clinic between January 2003 and December 2010. None of these patients had a disease or anatomic abnormality that would exacerbate GER. Dilatation treatment was applied to all patients with rigid dilators. Antacid medical treatment was sustained during dilatation treatment.

Gastro-oesophageal reflux is defined as either having abnormal findings in oesophageal $\mathrm{pH}$ monitoring (reflux index 4.2\%) or having endoscopic findings of oesophagitis. Single use monocrystal antimony $\mathrm{pH}$ electrode probe with dual sensors was used for $\mathrm{pH}$ measurements of the oesophagus and stomach (Synetics Medical, Queluz, Portugal). An ambulatory $\mathrm{pH}$ measurement device was used for recording $\mathrm{pH}$ values (Orion II, Medical Measurement Systems MMS, Enschede, Netherlands). The placement of the electrode was controlled and confirmed via direct $\mathrm{x}$-ray radiography. The proximal tip of the electrode was placed at three vertebral levels above the diaphragm and the distal tip was placed in the stomach. Acid reflux in distal oesophagus was evaluated by the following five parameters: reflux index, the total number of reflux episodes, the number of reflux episodes exceeding five minutes, the longest reflux period, and clearance time. Oesophageal $\mathrm{pH}$ monitoring was performed once for each patient, at $33.41 \pm 19.14$ months after the injury. Oesophageal stricture and the presence of oesophagitis according to the gross appearance of mucosa were assessed via flexible pediatric gastroscopy (Fujinon, FG-1 ZP, New Jersey, USA) using 3-6 month intervals during the two years of dilatation treatment.

Groups with and without GER were compared to each other in terms of the following: demographics, type of ingested substance, clinical symptoms of GER, results of ambulatory $\mathrm{pH}$ monitoring, frequency of GER, existence of Barrett's oesophagus, length of stricture, number of strictures, localisation of strictures, duration of dilatation treatment and success of treatment.

The localisation and number of strictures were determined by oesophagography and endoscopy. The lengths of strictures were noted during endoscopy using the scale on the endoscope. Since the length of the injured segment of oesophagus may have an impact on developing GER, patients were divided into two groups regarding their stricture lengths. Strictures shorter than $4 \mathrm{~cm}$ in length were assessed as "short", while those with a length equal to or larger than $4 \mathrm{~cm}$ were assessed as "long" strictures. The time period of benefit from dilatation was defined as the time between the first date of dilatation and at least three weeks after the last dilatation in which solid and liquid food were swallowed easily. Successful dilatation treatment was defined as having no dysphagia and no repetitive dilatation treatments at least one year after the last dilatation.

\section{Statistical analysis}

The Kolmogorov-Smirnov test was used to determine the normal data distribution when comparing groups with and without reflux. The distribution of these data generally failed to comply with the normal distribution. The Mann-Whitney U-test was used to compare these parameters. Chi-square test and logistic regression were used to analyse whether the reflux status of patients was related to the investigated parameters. A p value below 0.05 was considered to be statistically significant. The SPSS 11.0 program (IBM, Newyork, USA) was used for statistical analysis.

\section{RESULTS}

Our study consisted of 22 female and 30 male patients. The mean age of our study population was $4.2 \pm 2.88$ (1-14) years. The ingested substance was alkali in 27 patients and acid in 25 patients. Gastro-oesophageal reflux was present in 33 patients $(63.5 \%)$. Demographic data of patients with and without GER were comparable $(p>0.05)$. Gastro-oesophageal reflux was diagnosed via oesophageal $\mathrm{pH}$ monitoring in 28 patients, in which 9 of them were also diagnosed with oesophagitis. The remaining 5 patients had significant oesophagitis and were diagnosed with reflux despite their normal oesophageal $\mathrm{pH}$ monitoring findings. Voice hoarseness, sensation of food stuck in the throat, regurgitation and retrosternal burn were significantly more common in patients with GER than in patients without $(\mathrm{p}<0.05)$ (Table 1$)$. Frequent lower respiratory tract infections, asthma, hoarseness and repetitive otitis media were never observed in patients without GER.

Logistic regression analysis revealed significant regression values for 2 (ingested substance and stricture length) out of the 4 determined risk factors $(\mathrm{p}<0.05)$. Among the patients with GER, $21(63.6 \%)$ ingested alkali, whereas only 6 patients in the group without GER ingested alkali (31.6\%). This difference was statistically significant $(\mathrm{p}<0.05)$. GER was found 1.62-times more often in strictures caused by alkaline sub-

TABLE 1. Symptoms observed in groups with or without reflux

\begin{tabular}{lccc}
\hline & $\begin{array}{c}\text { Reflux (+) } \\
\text { Reflux (-) }\end{array}$ & (n) & Total \\
\hline Hoarseness & $6^{*}$ & 0 & 6 \\
Sensation of object stuck in the throat & $15^{*}$ & 2 & 17 \\
Regurgitation & $18^{*}$ & 3 & 21 \\
Retrosternal burn & $22^{*}$ & 2 & 24 \\
Recurrent lower respiratory tract infection & 1 & 0 & 1 \\
Cough & 5 & 1 & 6 \\
Halitosis & 25 & 11 & 36 \\
Hiccup & 5 & 3 & 8 \\
Rumination & 19 & 6 & 25 \\
Asthma & 1 & 0 & 1 \\
Recurrent bronchitis & 5 & 3 & 8 \\
Recurrent otitis media & 2 & 0 & 2 \\
Growth retardation & 9 & 5 & 14 \\
Nutrition denial & 2 & 1 & 3 \\
Dysphagia & 9 & 2 & 11 \\
\hline
\end{tabular}

${ }^{*} \mathrm{p}<0.05$ compared to reflux (-) group (Chi-square test) 
stances than strictures caused by acid substances (Odds Ratio $=1.62$ ). Thirty patients had a single stricture localisation and 22 patients had two separate strictures (Table 2). In patients without GER, $12(63.1 \%)$ out of 19 had a single stricture. In patients with GER, 18 (54.5\%) out of 33 patients had a single stricture. This difference was not statistically significant ( $p>0.05$ ). Additionally, the prevalence of GER did not differ significantly in terms of localisation of the stricture. $(\mathrm{p}>0.05)$.

When evaluating stricture length, 35 of the strictures were short and 17 were long (Table 2). Sixteen patients (48.5\%) in the GER group and only one patient (5.9\%) in the group without GER had long stricture. All six of the patients who had a stricture length over $5 \mathrm{~cm}$ were diagnosed with GER. The mean stricture length was $2.2 \pm 1.0 \mathrm{~cm}$ in patients without GER and $3.7 \pm 1.8 \mathrm{~cm}$ in patients with GER. There was a statistically significant difference between the two groups in terms of stricture length $(\mathrm{p}<0.005)$. Patients with long stricture were 1.9-times more likely to have GER than patients with short stricture (Odds Ratio=1.94).

When we compared the data obtained from oesophageal $\mathrm{pH}$ monitoring in patients with GER according to the length of stricture, none of the five parameters differed significantly between patients with long stricture and patients with short stricture (Table 3). Four (23.5\%) out of seventeen patients with short stricture were diagnosed with oesophagitis via endoscopy, whereas ten $(62.5 \%)$ out of sixteen patients with long stricture were diagnosed with esophagitis. This difference was statistically significant $(p<0.005)$. In the GER group, 14 of the 16 patients with long stricture had five or more symptoms and 10 out of 17 patients with short stricture had four or more.

During the first 3 months of observation, the mode value of the widest diameter of dilatator used during dilatation was $30 \mathrm{Fr}$ in patients with GER and $36 \mathrm{Fr}$ in patients without GER (Table 4). After the first 3 months, the diameters of dilatators used in both groups were similar. Diameters of dilatators used in the dilatation process did not differ significantly between the two groups.

Dilatation treatment was completed in 15 (78.9\%) of 19 patients without GER and in $23(69.69 \%)$ of 33 patients with GER. One patient from each group was referred to our hospital after the end of treatment with symptoms of recurrent stricture. There was no statistically significant difference between patients with GER and without GER in terms of success of treatment and need for repetitive dilatation. Thirty eight patients were treated successfully and no differences were observed between these patients in terms of treatment period, follow-up period, period benefited from dilatation and presence of GER ( $p>0.05$ ) (Table 5). A mean time of $44.40 \pm 22.16$ months passed from the beginning of the treatment of the 14 patients who had still been receiving treatment. Dilatation treatment was unsuccessful due to the inability to establish an oesophageal lumen and oesophageal replacement surgery was required in one of these patients.
TABLE 2. The numbers, localisation and length of strictures in groups with or without reflux

\begin{tabular}{llccc}
\hline $\begin{array}{l}\text { Characteristics } \\
\text { of stricture }\end{array}$ & & $\begin{array}{c}\text { Reflux }(+) \\
(\mathrm{n})\end{array}$ & $\begin{array}{c}\text { Reflux (-) } \\
(\mathrm{n})\end{array}$ & Total \\
\hline Localization & Proximal & 5 & 4 & 9 \\
& Mid & 21 & 11 & 32 \\
\multirow{4}{*}{ Number } & Distal & 7 & 4 & 11 \\
& 1 & 18 & 12 & 30 \\
Length & 2 & 15 & 7 & 22 \\
& Short $(<4 \mathrm{~cm})$ & $17^{*}$ & 18 & 35 \\
& Long $(\geq 4 \mathrm{~cm})$ & $16^{*}$ & 1 & 17 \\
\hline
\end{tabular}

$* \mathrm{p}<0.05$ compared to reflux (-) group (Chi-square test)

TABLE 3. Oesophageal $\mathrm{pH}$ monitoring data according to the length of stricture in groups with or without reflux

\begin{tabular}{lcc}
\hline & $\begin{array}{c}\text { Short stricture }(<4 \mathrm{~cm}) \\
\text { Mean } \pm \text { SD } \\
(\text { Min-Max) }\end{array}$ & $\begin{array}{c}\text { Long stricture }(\geq 4 \mathrm{~cm}) \\
\text { Mean } \pm \text { SD } \\
(\text { Min-Max })\end{array}$ \\
\hline The number of reflux period & $86.8 \pm 66.7$ & $79.3 \pm 54.3$ \\
& $(9.4-243.7)$ & $(21.8-231.8)$ \\
Reflux index (\%) & $12.6 \pm 14.9$ & $13.1 \pm 16.3$ \\
The number of reflux episodes & $(1.3-63.3)$ & $(1.3-64.0)$ \\
exceeding five minutes & $5.4 \pm 7.2$ & $4.3 \pm 5.7$ \\
The longest reflux episode (min) & $(0.0-28.1)$ & $(0.0-21.7)$ \\
& $19.3 \pm 22.8$ & $22.8 \pm 23.2$ \\
Clearance time (min) & $(2.5-74.0)$ & $(1.9-68.1)$ \\
& $1.6 \pm 1.4$ & $1.4 \pm 0.9$ \\
& $(0.6-6.2)$ & $(0.6-3.5)$ \\
\hline
\end{tabular}

Differences between the groups were not statistically significant (Mann-Whitney U-test)

TABLE 4. The widest dilatator diameter used in treatment of groups with or without reflux

\begin{tabular}{lcc}
\hline Time period & $\begin{array}{c}\text { Reflux }(+) \\
\text { Mode } \\
\text { (Min-Max) }\end{array}$ & $\begin{array}{c}\text { Reflux }(-) \\
\text { Mode } \\
\text { (Min-Max) }\end{array}$ \\
\hline 0-3 months & 30 & 36 \\
3-6 months & $(24-45)$ & $(15-45)$ \\
& 36 & 36 \\
6-9 months & $(27-45)$ & $(24-45)$ \\
9-12 months & 45 & 42 \\
& $(27-45)$ & $(33-45)$ \\
& 45 & 45 \\
& $(30-51)$ & $(36-45)$ \\
\hline
\end{tabular}

Differences between the groups were not statistically significant (Mann-Whitney U-test)

\section{DISCUSSION}

The most important complication of oesophageal injury related with corrosive ingestion is CES which occurs in 25-65\% of patients $(1,2)$. The success rate of the standard oesophageal dilatation treatment is reported to be approximately $50-70 \%$, but there is some variability between series (1-4). The effects of referral time, type and amount of the substance ingested, degree of injury, initiation time of the dilatation treatment 
TABLE 5. The relationship between treatment, follow-up periods and reflux in patients who had their treatment completed

\begin{tabular}{lcc}
\hline Time period & Reflux (+) & Reflux (-) \\
& Mean \pm SD & Mean \pm SD \\
& (Min-Max) & (Min-Max) \\
\hline Period benefited from dilatation (months) & $8.41 \pm 6.1$ & $8.21 \pm 8.4$ \\
& $(1-24)$ & $(1-30)$ \\
Treatment period (months) & $35.74 \pm 22.0$ & $33.31 \pm 19.7$ \\
Follow-up period (months) & $(4-84)$ & $(9-85)$ \\
& $21.2 \pm 17.8$ & $20.4 \pm 21.6$ \\
& $(0-59)$ & $(1-68)$ \\
\hline Differences between the groups were not statistically significant(Mann-Whitney U-test)
\end{tabular}

and length of stricture are clearly demonstrated in studies performed to investigate the causes of these low and variable success rates (5-9).

Recent studies have shown that another factor which has affected treatment success rates is GER $(4,7,10)$. Patients with CES have a recovering lesion that occurs because of burn injury. Previous researchers have hypothesised that fibrosis, formed during recovery of injury in the lesion, will not only cause stricture of the lumen in the horizontal plane but will also cause shortening of oesophageal length by contraction in the vertical plane $(6,7,11)$. Although this hypothesis has been put forward, the number of studies researching the prevalence of GER in patient groups with CES is limited (5-9). We performed a literature review of studies published in English and found a total of five articles which demonstrated that prevalence of GER in cases with CES varies between 58-76\%. Despite such high prevalence rates being reported, factors affecting GER occurrence and their effects on CES treatment were not discussed in any of these studies. Investigation of possible factors affecting GER occurrence and their effects on stricture treatment is one of the original findings of our study in which the prevalence of GER was found to be similar to that in other studies.

There are several different research methods and approaches in the diagnosis of GER. Bautista et al. (8) have found a higher prevalence of oesophagitis in patients with CES. In our study, one third (14 of 52) of patients with CES had oesophagitis. GER could be diagnosed with the presence of oesophagitis in our five patients. Only two previous studies accounted for findings of endoscopic oesophagitis when investigating the presence of GER in CES $(5,9)$. In both of these studies, as in our study, there was a group of patients that could only be diagnosed via endoscopy. There was a similar situation when studying the presenting symptoms. We found that the patients with GER experienced symptoms, especially voice hoarseness, the sensation of something stuck in the throat, regurgitation and retrosternal burn, more frequently. We suggest considering both endoscopic oesophagitis and GER symptoms when investigating GER in patients with CES rather than using oesophageal $\mathrm{pH}$ monitoring as a sole measurement.
Da-Costa-Pinto et al. (5) suggested that GER is due to both shortening of oesophageal length in the vertical plane because of fibrosis and motility disorder secondary to corrosive substance injury in patients with CES. An objective diagnostic method showing shortening of oesophageal length could not be defined. In patients experiencing oesophageal replacement and oesophagectomy because of CES, extensive fibrosis is mainly observed in mucosal, submucosal and inner muscular layers in histopathological examinations (5).

On the other hand, Da-Costa-Pinto et al. (5) found motility disorders in these patients and asserted that the motility disorder occurs due to the restrictive effect of fibrosis in the muscular layer as the myenteric plexus was found to be intact in oesophageal tissues. Genç et al. (11) have shown that motility disorders continue even when the dilatation treatment is successful. In this instance, it is obvious that both of the two suggested contributing factors (shortening of oesophageal length and motility disorder) in the pathophysiology of GER in CES are related to the severity and length of fibrosis.

Regardless of the mechanisms of GER, there is an ongoing healing process of the injured oesophagus due to dilatation treatment in CES patients (12). Thus, GER might aggravate the fibrosis on corrosive stricture, in the same manner as peptic oesophagitis. When this hypothesis is taken into account, it has been suggested that both the shortening of oesophageal length will cause GER and GER will have a negative effect on the treatment of stricture, as in cases of oesophageal atresia. This vicious circle type of relation should be enlightened in prospective studies with larger series of long stricture patients.

We evaluated four risk factors of GER development in CES patients. We observed that besides stricture, the incidence of GER was observed 1.6-times more often in burns occurring with alkali than with acid substances. Since the relationship between type of substance ingested and GER incidence has not been investigated in other studies, it was not possible to compare our findings. Our study is unique because of this. Similarly, we could not find a study investigating the relationship between GER and the number and length of stricture in CES. In our study, even though GER prevalence was found to be slightly higher in patients with more than one stricture compared with patients having single stricture; the difference was not significant. We can conclude that number of strictures does not influence GER occurrence. While almost all of the patients with strictures longer than $4 \mathrm{~cm}$ also had GER, only half of those with shorter strictures had GER. This shows that length of stricture has a close relationship with GER and is an important factor in its presence. To define whether length of stricture had an effect on GER severity or not, we compared data of oesophageal $\mathrm{pH}$ monitoring, prevalence of symptoms and oesophagitis in patients with longer and shorter strictures. We found the prevalence of both symptoms and oesophagitis to be evidently higher in the patient group with longer stric- 
tures. Thus, we believe that longer strictures cause more severe GER. It may be that CES involving the distal oesophagus affects LES function negatively and causes GER more often. Da-Costa-Pinto et al. (5) studied oesophageal functions in patients with CES and GER prevalence did not differ according to localisation of the stricture in their studies. In this study, even though the population of patients with distal strictures was relatively larger and lengths of strictures were longer, it was remarkable to find that GER prevalence was not higher in this group of patients and that LES pressure was normal in many of them. As we did not observe a higher prevalence of GER in CES patients with distal strictures, we can conclude that there is no relationship between location of the stricture and GER prevalence.

Capella et al. (10) were the first to draw attention to the effect of GER on dilatation treatment. Dilatation treatment could be successfully completed after anti-reflux surgery in all four patients, which indicates that GER has a negative effect on CES treatment. Of the studies questioning the relationship between CES and GER, the effect of GER on dilatation treatment is only discussed in the study of Mutaf et al. (6). In this study, GER prevalence was 66\% (35/53) and researchers investigated treatment responses based on the reflux index obtained from oesophageal $\mathrm{pH}$ monitoring. They defined unsuccessful treatment in all of the patients with a reflux index of more than 20. In contrast, in patients with reflux index lower than 20 , treatment success was found to be close to $90 \%$. The details of the treatments were not discussed in any of the studies, including that of Mutaf et al. (6).

We investigated parameters such as dilator diameters, treatment period, and the persistence of symptoms after the end of treatment in order to identify the effects of GER on treatment in our study. Mode values of dilator diameters used in the first three months throughout the first year following the introduction of dilatation did not show any difference between patient groups with or without GER. In our series, our overall treatment success rate is $73 \%$ in patients with CES. In one of our cases, colonic interposition was required. The current treatment of the remaining patients is ongoing. In patients who completed treatment, comparison in terms of GER diagnosis did not show a significant difference between the two groups. Due to all of these findings related to treatment, we concluded that GER did not affect the treatment success of our patients in the short-term. In patients who completed treatment, followup periods were similar and the beneficial time from dilatation treatment and treatment periods showed no difference between the patient groups. These findings obtained from those patients who completed treatment support our opinion that GER is not affecting the course of dilatation treatment. In this regard, one of the important questions to study is the timing of anti-reflux surgery in CES patients who have been diagnosed with GER. It was observed that GER did not have an evident effect on standard dilatation treatment and the completion time of dilatation treatment was similar between patients with or without GER. As a result of these findings, we would consider delaying the intervention in patients requiring anti-reflux surgery to a period of time once it is decided that dilatation treatment is successful.

There are some limitations of our study. The fact that it was a retrospective study is the most important limitation. A control group of healthy children was not established due to ethical concerns. We only performed a GER survey once and it was during dilatation treatment; this may be an obstacle in both the evaluation of long-term trends of GER and harmful effects of dilatation in terms of GER.

We investigated GER by using more than one method systematically in patients with corrosive oesophageal stricture. Our findings revealed that CES causes GER in high extents. Of the risk factors of GER occurrence in CES patients, only alkali substance ingestion and length of the stricture have significant relationship with GER. Since two-thirds of our patients were symptomatic and half of them had oesophagitis, this shows that GER has an evident clinical effect on this group of patients. For this reason, we suggest GER investigation for all patients having CES during the dilatation treatment. We also recommend prospectively evaluating the effects of GER in a large number of patients.

Ethics Committee Approval: Ethics committee approval was received for this study from the ethics committee of Çukurova University. Informed Consent: Written informed consent was obtained from patients'
parents who participated in this study.

Peer-review: Externally peer-reviewed.

Author contributions: Concept - S.H.İ., Z.Ö.; Design - S.H.İ., Z.Ö.; Supervision - S.H.İ., Ü.Z.; Resource - S.H.İ., M.A., S.T.; Materials - Z.Ö., S.T.; Data Collection\&/or Processing - Z.Ö., S.T.; Analysis\&/or Interpretation - S.H.I., Z.Ö., Ü.Z.; Literature Search - Z.Ö., M.A.; Writing - S.H.İ., Z.Ö.; Critical Reviews - S.H.İ., Z.Ö., M.A., S.T., Ü.Z.

Acknowledgements: We want to thank to Nazif Çalış who helped us for the assessment of statistical data and contributed to our study.

Conflict of Interest: No conflict of interest was declared by the authors.

Financial Disclosure: The authors declared that this study has received no financial support.

\section{REFERENCES}

1. Erdoğan E, Eroğlu E, Tekant G, Yeker Y, Emir H, Sarımurat N, et al. Management of esophagogastric corrosive injuries in children. Eur $J$ Pediatr Surg 2003;13:289-93. [CrossRef]

2. Gün F, Abbasoğlu L, Çelik A, Salman FT. Early and late term management in caustic ingestion in children: a 16-year experience. Acta Chir Belg 2007;107:49-52. 
3. Chang CF, Kuo SP, Lin HC, Chuang CC, Tsai TK, Wu SF, et al. Endoscopic balloon dilatation for esophageal strictures in children younger than 6 years: experience in a medical center. Pediatr Neonatol 2011;52:196-202. [CrossRef]

4. Panieri E, Rode H, Millar AJW, Cywes S. Oesophageal replacement in the management of corrosive strictures: when is surgery indicated? Pediatr Surg Int 1998;13:336-340. [CrossRef]

5. Da-Costa-Pinto EAL, Dorsa TK, Altimani A, Andreollo NA, Cardoso SR, Morais DJ, et al. A functional study of caustic strictures of the esopagus in children. Braz J Med Biol Res 2004;37:1623-30. [CrossRef]

6. Mutaf O, Geç A, Herek Ö, Demircan M, Özcan C, Arikan A. Gastroesophageal reflux: a determinant in the outcome of caustic esophageal burns. J Pediatr Surg 1996;31:1494-5. [CrossRef]

7. Özcan Z, Özcan C, Erinç R, Dirlik A, Mutaf O. Scintigraphy in the detection of gastroesophageal reflux in children with caustic oesophageal burns: a comparative study with radiography and 24-h pH monitoring. Pediatr Radiol 2001;31:737-41. [CrossRef]

8. Bautista A, Varela R, Villanueva A, Estevez E, Tojo R, Cadranel S. Motor function of the esophagus after caustic burn. Eur J Pediatr Surg 1996;6:204-7. [CrossRef]

9. Bautista A, Martinez EE, Cives RV, Jeremias AV, Tojo R, Cadranel S. A retrospective analysis of ingestion of caustic substances by children. Ten-year statistics in Galicia. Eur J Pediatr 1997;156:410-4. [CrossRef]

10. Capella M, Goldberg P, Quaresma E, Araujo E, Pereime M. Persistance of corrosive esophageal stricture due to gastroesophageal reflux in children. Pediatr Surg Int 1992;7:180-2.[CrossRef]

11. Genç A, Mutaf O. Esophageal motility changes in acute and late periods of caustic esophageal burns and their relation to prognosis in children. $J$ Pediatr Surg 2002;37:1526-8. [CrossRef]

12. Dantas RO, Mamede RC. Esophageal motility in patients with esophageal caustic injury. Am J Gastroenterol 1996;91:1157-61. 